\title{
A Forward-Secure Signature with Backward-Secure Detection
}

\author{
Dai-Rui Lin and Chih-I Wang \\ Department of Computer Science and Engineering \\ National Sun Yat-sen University, Kaohsiung 804, Taiwan
}

June 17, 2007

\begin{abstract}
This paper enhances the security of Abdalla and Reyzin's forwardsecure signature scheme with backward-secure detection. In the proposed scheme, we embeded the hash-chain into the forward-secure signature scheme. It achieves not only forward-secure but also backwardsecure for the digital signature.
\end{abstract}

Keywords : Forward-Secure, Backward-Secure, Digital signatures, GQ signatures.

\section{Introduction}

The key exposures carries very serious problems, it is regarded a total break of the system. In order to avoid this undesirable situation, the goal of forward security is to protect against this kind of threat.

A protocol is said to provide forward secrecy if the compromise of longterm keys does not compromise past session keys that have been established before the compromise of the long-term key [3]. Bellare and Miner first proposed signatures with forward-security properties [3]. In 2001, Abdalla and Reyzin improved the Bellare-Miner's forward-secure GQ signature schemes with a shorter public key [2]. Since then, many works about forward-secure related schemes has been proposed $[8,5,6,1,9,10,7]$.

The main concept of forward-secure signature scheme is that: the public key is fixed but the secret signing key is updated at regular intervals. Each secret signing key are use for sign messages only during a particular time period. At the end of each time period, a new secret key is produced and 
old one is erased. This can be useful to mitigate the damage caused by key exposure without requiring distributions of keys.

However, once the key is exposed, the security of past uses of the keys can be protected, but the future uses of it are compromised. That is, once the future secret keys can be forge, even the original signer cannot detect the secret keys have been used, it is regarded a total crash of the system. Therefore, the perfect solution is that intrusion is detected and we change keys.

In this paper, we improve the Abdalla-Reyzin's forward-secure signature scheme with backward-secure detection. Our constructions achieve not only forward-secure but also backward-secure for the digital signature. We employ the concept of hash-chain to the digital signature. This can ensure the security of the signature be more robust and achieve that the future signature cannot be forge even the secret key are exposed.

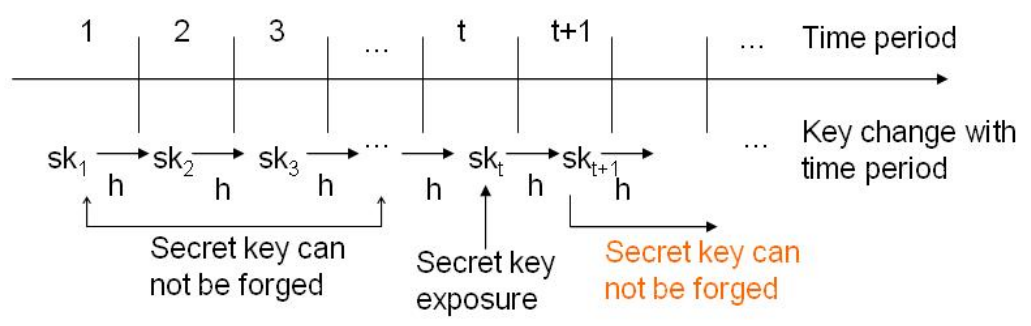

Figure 1: Forward-security and backward-security

\section{Review of Abdalla-Reyzin's Forward-secure GQ signature scheme}

In this section, we will brief review of forward-secure GQ signature scheme that proposed by Abdalla and Reyzin [2]. Abdalla-Reyzin's was improve the Bellare-Miner's forward-secure GQ signature schemes [3]. Abdalla-Reyzin's schemes has significantly shorter keys that more practical then Bellare-Miner's schemes and depicted in Figure 1. Their scheme has also be proven forward secure in the random oracle model, assuming factoring is hard. The scheme is divided into four phases, (1) key generation, (2) signature generation, (3) signature verification and (4) key updating.

- Key generation phase:

Let $p \equiv q \equiv 3(\bmod 4)$ be two primes and $N=p q$ be a $k$-bit integer. The signer chooses a random number $s_{0} \in Z_{N}^{*}$ as his/her signing 
key, and computes the corresponding verification key $u=1 / s_{0}^{2^{l(T+1)}}$ $\bmod N$, where $T$ denotes as the total number of periods, and $l$ denotes as the length of binary string. The signer publishes the verification key set $V K=(N, u, T)$.

- Signature generation phase:

Then the signer generate the signature for period $j$ by the $j$ th period secret key $s_{j}$ as follows. First, he/she chooses a random number $r \in Z_{N}^{*}$ and computes $a=H(j, y, M)$ and $z=r\left(s_{j}\right)^{a} \bmod N$, where $y=$ $r^{2^{l(T+1-j)}} \bmod N$, and $M$ is the message to be signed. The signature for period $j$ is $(j, a, z)$ and publish $(j, a, z, M)$ to verifier.

- Signature verification phase:

Upon receiving $(j, a, z, M)$, the verifier computes $y^{\prime}$ as $y^{\prime}=z^{2^{l(T+1-j)}} u^{a}$ $\bmod N$. Then, he computes $a^{\prime}$ as $a^{\prime}=H\left(j, y^{\prime}, M\right)$. The signature is valid if $a=a^{\prime}$.

- Key updating phase:

Via key-updating mechanism, in each current period $j$, the signer updating the $j$ th period signing key $s_{j}$ into $s_{j+1}$ as $s_{j+1}=s_{j}^{2^{l}} \bmod N$. The public key stays the same.

\begin{tabular}{|c|c|c|}
\hline Sender & & Receiver \\
\hline$s_{j}=s_{j-1}^{2^{l}} \bmod N$ & & $\begin{array}{l}y^{\prime}=z^{2^{l(T+1-j)}} u^{a} \bmod N \\
=\left(r s \cdot^{a} 2^{2^{l(T+1-j)}} u^{a} \bmod N\right.\end{array}$ \\
\hline$y=r^{2^{l(T+1-j)}} \bmod N$ & $j, a, z, M$ & $=\left(r s_{j}{ }^{a}\right)^{2^{l(T+1-j)}} 1 / s_{0}^{2^{l(T+1)} a} \bmod N$ \\
\hline$a=H(j, y, M)$ & & $=r^{2^{l(T+1-j)}} \bmod N$ \\
\hline$z=r s_{j}{ }^{a} \bmod N$ & & $a \stackrel{?}{=} H\left(j, y^{\prime}, M\right)$ \\
\hline
\end{tabular}

Fig. 2. The Abdalla-Reyzin's forward-secure GQ signature.

\section{Forward-secure signature with backward- secure detection}

In this section, we consider the backward-secure of Abdalla-Reyzin's forwardsecure signature. We implement a backward-secure on the condition that the verification formula of the underlying signature scheme is kept unchanged. 


\subsection{The one-way hash chain}

Definition 1. Let $h(\cdot)$ be a collision-resistant one-way hash function, we denote the $h^{T}(\cdot)$ be a one-way hash chain, where $h^{T}(\cdot)=h\left(h^{T-1}(\cdot)\right)=h\left(h\left(h^{T-2}\right.\right.$ $(\cdot)))=\overbrace{h(h \ldots(h(}^{T} \cdot)) \ldots)$ and $h^{0}(\cdot)=\cdot$. The $T$ be the total number of time periods.

In order to achieve the forward-secure signature with detection of backwardsecure, during the period $j$, by employee the hash function $h$, the $j$ th random number $r_{j}$ can define as $r_{j}=h^{T-j}(x)$, where $x$ is the random number or the personal secret password.

\subsection{Definitions}

We denoted the propose schemes by FSBD, is integrate hash chain technique into forward-secure signature. The propose schemes consists of five procedures.

Definition 2. A FSBD consists of five algorithms that $\mathbf{F S B D}=\left(\mathbf{F S B D} \cdot{ }_{\text {keyGen }}\right.$, FSBD.sign, FSBD.verify, FSBD.backDet, FSBD.keyUpdate):

- Key generation algorithm FSBD.keyGen: it is a probabilistic polynomialtime algorithm that takes as input a security parameter $k$ and possibility other parameters and returns a public key $u$ and corresponding private key $s_{0}$. That is, $\mathbf{F S B D}_{\mathbf{k n y G e n}}\left(1^{k}\right)=\left(u, s_{0}, l\right)$, where $l$ is the system secret.

- Signature generation algorithm FSBD.sign: the signing algorithm, takes as input the secret key $s_{j}$ for the time period $j(j \leq T)$ and the message $M$ to be signed and returns the signature $<j$, sign $>$ of $M$ for time period $j$. That is, $\mathbf{F S B D} \cdot \operatorname{sign}\left(j, s_{j}, M\right)=($ sign $)$.

- Signature verification algorithm FSBD.verify: the verification algorithm, takes as input the public key $u$, a message $M$, and a candidate signature $<j$, sign $>$. The algorithm will return 1 if $\langle j$, sign $\rangle$ is a valid signature of $M$. Otherwise, it will return 0 . That is,

$\operatorname{FSBD}_{\text {verify }}(j, u, M, \operatorname{sign})=1$.

- Backward detection algorithm FSBD.backDet: the backward detection algorithm, takes as input the $j+1$-th value $r_{j+1}$, computes $r_{j}$ from $h\left(r_{j+1}\right)$ as $r_{j}=h\left(r_{j+1}\right)=h^{T-j}(x)$. The algorithm will return 1 if FSBD.backDet $\left(j, r_{j+1}, y_{j}^{\prime}\right)=1$, where $y_{j}^{\prime}=r_{j}^{2^{l(T+1-j)}} \bmod N$. Otherwise, it will return 0 . 
- Key updating algorithm FSBD.keyUpdate: the secret key update algorithm, takes as input the secret key $s_{j}$ for the current period $j<T$ and returns the new secret key $s_{j+1}$ for the next period $j+1$. That is, $\operatorname{FSBD}_{\text {keyUpdate }}\left(j, s_{j}\right)=\left(s_{j+1}\right)$.

Definition 3. A forward secure signature with the properties of backwardsecure and backward-secure detection if the compromise of secret key $s_{i}$ will not compromise the $s_{j}$ for all $j>i$, and the verifier can detect the signature has been forge or not by the hash chain value $h^{i+1}(x)$ for each time period $i>0$.

Definition 4. (The Blum Factorization Problem) Given n, where $n$ is the product of two distinct large primes $p$ and $q$ with roughly the same length $p \equiv q \equiv 3(\bmod 4)$, find $p$ or $q$.

\subsection{The proposed scheme}

Our construction of the proposed scheme depicted in Figure 2. The proposed scheme divided into five phases, (1) key generation, (2) signature generation, (3) signature verification, (4) backward-secure detection and (5) key updating. We describe the details of the five phases as follows.

- Key generation phase:

The proposed scheme consists two keys and one hash chain number, the signing $\operatorname{key}(s)$, the verification $\operatorname{key}(u)$, and the hash chain number $\left(r_{0}\right)$. Let $p \equiv q \equiv 3(\bmod 4)$ be two primes and $N=p q$ be a $k$-bit integer. The signer chooses a random number $s_{0} \in Z_{N}^{*}$ as his/her signing key, and computes the corresponding verification key $u=1 / s_{0}^{2^{l(T+1)}}$ $\bmod N$, where $T$ denotes as the total number of periods, and $l$ denotes as the length of binary string. The signer publishes the verification key set $V K=(N, u, T)$. Finally, the signer random choose a random number $x \in Z_{N}^{*}$ and computes $r_{0}=h^{T}(x)$.

- Signature generation phase:

Then the signer generate the signature for period $j$ by the $j$ th period secret key $s_{j}$ as follows. First, he/she computes the $j$ th and the $j+1$ th hash chain number $r_{j}$ and $r_{j+1}$ as $r_{j}=h^{T-j}(x)$ and $r_{j+1}=$ $h^{T-j+1}(x)$, then he/she computes $a_{j}=H\left(j, r_{j+1}, y_{j}, M_{j}\right)$ and $z_{j}=$ $r_{j}\left(s_{j}\right)^{a_{j}} \bmod N$, where $y_{j}=r_{j}^{2^{l(T+1-j)}} \bmod N$, and $M_{j}$ is the $j$ th message to be signed. The signature for period $j$ is $\left(j, r_{j+1}, a_{j}, z_{j}, M_{j}\right)$ and publish $\left(j, r_{j+1}, a_{j}, z_{j}, M_{j}\right)$ to verifier. 
- Signature verification phase:

Upon receiving $\left(j, r_{j+1}, a_{j}, z_{j}, M_{j}\right)$, the verifier computes $y_{j}^{\prime}$ as $y_{j}^{\prime}=$ $z_{j}^{2^{l(T+1-j)}} u^{a_{j}} \bmod N$. Then, he computes $a_{j}^{\prime}$ as $a_{j}^{\prime}=H\left(j, y_{j}^{\prime}, M_{j}\right)$. The signature is valid if $a_{j}=a_{j}^{\prime}$.

- Backward detection phase:

After verify the signature in regular process, he/she can use the $r_{j+1}$ to detect the secret key has been expose or not as follows. First, the verifier computes $r_{j}$ as $r_{j}=h\left(r_{j+1}\right)$, where $r_{j} \equiv h\left(h^{T-(j+1)}(x)\right) \equiv$ $h^{T-j}(x)$, then the verifer check that $y_{j}^{\prime} \stackrel{?}{=}\left(h^{T-j}(x)\right)^{2^{l(T+1-j)}}$

- Key updating phase:

Via key-updating mechanism, in each current period $j$, the signer updating the $j$ th period signing key $s_{j}$ into $s_{j+1}$ as $s_{j+1}=s_{j}^{2^{l}} \bmod N$. The public key stays the same.

\begin{tabular}{|c|c|c|}
\hline $\begin{array}{l}\text { Signer } \\
s_{0} \in_{R} Z_{N}^{*} \\
u=1 / s_{0}^{2^{l(T+1)}}(\bmod N) \\
s_{j}=s_{j-1}^{2^{l}}(\bmod N) \\
r_{j}=h^{T-j}(x) \\
r_{j+1}=h^{T-(j+1)}(x) \\
y_{j}=r_{j}^{2^{l(T+1-j)}}(\bmod N) \\
a_{j}=H\left(j, y_{j}, M_{j}\right) \\
z_{j}=r_{j}\left(s_{j}\right)^{a_{j}}\end{array}$ & $\stackrel{j, r_{j+1}, a_{j}, z_{j}, M_{j}}{\longrightarrow}$ & $\begin{array}{l}\text { Verifier } \\
y_{j}^{\prime}=z_{j}^{2^{l(T+1-j)}} u^{a_{j}}(\bmod N) \\
=\left(z_{j}\right)^{2^{l(T+1-j)}}\left(1 / s_{0}^{2^{l(T+1)}}\right)^{a_{j}} \\
=\left(r_{j}\left(s_{j}\right)^{a_{j}}\right)^{2^{((T+1-j)}}\left(1 / s_{0}^{2^{l(T+1)}}\right)^{a_{j}} \\
=r_{j}^{2^{l(T+1-j)}} s_{0} a_{j} 2^{l(T+1)}\left(1 / s_{0}^{2^{l(T+1)}}\right)^{a_{j}} \\
=r_{j}^{2^{l(T+1-j)}}(\bmod N) \\
a_{j} \stackrel{?}{=} H\left(j, y_{j}^{\prime}, M_{j}\right) \\
\text { backward-secure detection } \\
r_{j}=h\left(r_{j+1}\right)=h^{T-j}(x) \\
\left(h^{T-j}(x)\right)^{2^{l(T+1-j)}} \stackrel{?}{=} y_{j}^{\prime}\end{array}$ \\
\hline
\end{tabular}

Fig. 3. The proposed scheme

\section{Security Analysis and Discussions}

It is straightforward that the security of the proposed scheme as equal to the forward-secure signature scheme as describe in [4] by Abdalla and Reyzin. They improve the forward-secure signature scheme in the random oracle model. In this section, we will show that the proposed scheme is robust that satisfy not only the forward secure, but also backward secure. The properties comparisons between our scheme and previous schemes [3, 10, 8, 9, 2] are shown in Table 1. 


\subsection{Correctness}

Theorem 2. (Correctness) Let $j, r_{j+1}, a_{j}, z_{j}, M_{j}$ be a signature values produced by the scheme of Section 3. The execution of our proposed scheme between the signer and the verifier is always successful.

Proof. From figure 2, we can see the correctness of our schemes is sound. First, the verifier computs $y_{j}^{\prime}$ from $\left(j, z_{j}, u, a_{j}, N\right)$ as $y_{j}^{\prime}=z_{j}^{2^{l(T+1-j)}} u^{a_{j}}(\bmod N)$. Then, the verifier check that $a_{j} \stackrel{?}{=} H\left(j, y_{j}^{\prime}, M_{j}\right)$. Finally, the verifier detect the backward-security as $\left(r_{j}\right)^{2^{l(T+1-j)}} \stackrel{?}{=} y_{j}^{\prime}$. If both of the two express is hold, then the signature is valid.

\subsection{Forward-seucre protection}

Forward-secrecy refers to that the compromise of one or several secret keys does not compromise previous secret keys. Each secret signing key is adopted to sign messages only during a particular time period. Assume that the $j+1$ th secret key exposed, an adversary $A$ success forged a $j$-th valid signature for a message $m$, however, $A$ can not forged the past uses of the secret keys, since computs $s_{j-1}$ from $s_{j}^{2^{-l}} \bmod N$ is irreversible, this is equivalent to factor $N$. Therefore, the security of past uses of the keys can be protected.

\subsection{Backward-secure protection and detection}

Backward-secrecy refers to that the compromise of one or several secret keys does not compromise future secret keys.

Theorem 1. (The Backward-secure Detection) If there exists an attacker $A$ who can break the $j$-th period in the random oracle model, and success gain a valid signature $\left(j, a_{j}, z_{j}\right)$ for message $M^{*}$. Then the FSBD.backDet procedure is backward-secure detection that against the malicious attacker.

Proof. Suppose an attacker $A$ success forged a valid signature $\left(j, a_{j}, z_{j}\right)$ for message $M^{*}$, however, $A$ can not success break FSBD.backDet procedure, since the $A$ has to provide the $j+1$-th hash value $r_{j+1}$ for computs $r_{j}\left(r_{j}=h\left(r_{j+1}\right)=h^{T-j}(x)\right)$, and the verifier has to check the $\left(r_{j}\right)^{2^{l(T+1-j)}} \stackrel{?}{=} y_{j}^{\prime}$. The attacker $A$ computs $r_{j}$ from $r_{j+1}$ is irreversible. Therefore, the backwardsecurity of future uses of the keys can be protected and detected, even the Blum Factorization Problem totally break. 
Table 1 . The comparisons between $[8,3,2,5,10,9]$ and our scheme.

\begin{tabular}{lllllllll}
\hline \hline & {$[8]$} & {$[3]$} & {$[2]$} & {$[5]$} & {$[10]$} & {$[9]$} & Our scheme \\
\hline \hline $\begin{array}{l}\text { Forward } \\
\text { secure }\end{array}$ & $\sqrt{ }$ & $\sqrt{ }$ & $\sqrt{ }$ & $\sqrt{ }$ & $\sqrt{ }$ & $\sqrt{ }$ & $\sqrt{ }$ \\
\hline $\begin{array}{l}\text { Backward } \\
\text { secure }\end{array}$ & $\times$ & $\times$ & $\sqrt{ }$ & $\sqrt{ }$ & $\sqrt{ }$ & $\sqrt{ }$ & $\sqrt{ }$ \\
\hline $\begin{array}{l}\text { Backward } \\
\text { detection }\end{array}$ & $\times$ & $\times$ & $\times$ & $\times$ & $\times$ & $\sqrt{ }$ & $\sqrt{ }$ \\
\hline
\end{tabular}

\section{Conclusions}

In this manuscript we have enhances the security of Abdalla and Reyzin's forward-secure digital signature scheme with backward-secure detection. Once the secret key is exposed, both of the security of past/future uses of the keys can be protected. We achieved that the intrusion of the future signature can be detected as we desired.

\section{References}

[1] M. Abdalla and M. Bellare. Increasing the lifetime of a key: a comparative analysis of the security of re-keying techniques. In Proc. ASIACRYPT '00, LNCS 1976, pages 431-448, 1999.

[2] M. Abdalla and L. Reyzin. A new forward-secure digital signature scheme. In Proc. ASIACRYPT '00, LNCS 2139, pages 116-129. Springer-Verlag, 2001.

[3] M. Bellare and S. K. Miner. A forward-secure digital signature scheme. In Proc. CRYPTO '99, LNCS 1666, pages 431-448. Springer-Verlag, 1999.

[4] S. Goldwasser, S. Micali, and R.L. Rivest. A digital signature scheme secure against adaptive chosen-message attacks. Technical report, MIT Lab., Computer Science, Cambridge, Mass., March, 1995.

[5] G. Itkis and L. Reyzin. Forward-secure signatures with optimal signing and verifying. In Proc. CRYPTO '01, LNCS 2139, pages 332-354. Springer-Verlag, 2001. 
[6] H. Krawczyk. Simple forward-secure signatures from any signature scheme. In Proceedings of the 7th ACM Conference on Computer and Communications Security, pages 108-115, 2000.

[7] C. F Lu and S. Shieh. Secure key-evolving protocols for discrete logarithm schemes. In CT-RSA 2002, LNCS 2271, pages 300-309, 2002.

[8] C. F. Lu and S. Shieh. Efficient key-evolving protocol for the gq signature. Journal of information science and engineering, 20:763-769, 2004 .

[9] W. G Tzeng and Z. J Tzeng. Robust forward-secure signature schemes with proactive security. In Proc. PKC '00, LNCS 1992, pages 264-276, 2001.

[10] W. G Tzeng and Z. J Tzeng. Robust key-evolving public key encryption schemes. In ICICS 2002, LNCS 2513, pages 61-72, 2002. 